\title{
MIP/MTMR14 and muscle aging
}

\author{
Scott K. Powers ${ }^{1}$ and Michael B. Reid ${ }^{2}$ \\ ${ }^{1}$ Department of Applied Physiology and Kinesiology, University of Florida, Gainesville, FL 32611, USA \\ ${ }^{2}$ Department of Physiology and Center for Muscle Biology, University of Kentucky, Lexington, KY 40511, USA
}

Commentary on: Romero-Suarez et al. Muscle-specific inositide phosphatase (MIP/MTMR14) is reduced by age and its loss accelerates skeletal muscle aging process by altering calcium homeostasis. Aging 2010; 2: 504-513.

E-mail: mbreid2@email.uky.edu

It is well known that the human aging process is associated with a progressive loss of muscular strength. Characteristic of this decline in muscle performance is the loss of skeletal muscle mass (sarcopenia) that occurs even in the healthy elderly. Indeed, humans can lose as much as $40 \%$ of their muscle mass from age 20 to 60 . This significant loss of muscle mass and strength has important health and social implications as muscle weakness can contribute to an increased risk of falls and a loss of independence. Therefore, improving our understanding of the mechanisms responsible for sarcopenia and the age-related loss of muscle strength is important.

The report by Romero-Suarez et al. [1] provides important new information regarding a potential cellular mechanism that may contribute to sarcopenia. These investigators used a combination of experimental approaches to phenotypically compare wild type old mice with mature mice lacking a novel muscle specific inositide phosphatase (MIP/MTMR14) that plays an important role in muscle calcium homeostasis. Interestingly, the mature MIP/MTMR14 knockout mice displayed phenotypes that closely resembled the muscles of old animals. Indeed, these relatively young knockout mice displayed impaired muscle calcium homeostasis, depressed muscle contractile force production and a loss of muscle mass that is commonly observed in senescent animals. Interestingly, the old wild type mice also displayed impaired muscle calcium homeostasis and decreases in muscle size and contractile function. Importantly, these old mice also possessed reduced muscle levels of MIP/MTMR14. The authors concluded that these findings were consistent with the hypothesis that an age-related loss in MIP/MTMR14 may be a contributory factor to sarcopenia.
The findings of Romero-Suarez et al. [1] are exciting and set the stage for future experiments to further examine the molecular role that MIP/MTMR14 plays in the age-related decline in skeletal muscle mass and function. Prior studies by Shen, et al. [2] suggest that MIP/MTMR14 deficiency elevates PtdIns(3,4)P2 and PtdIns(3,5)P2 levels in the sarcoplasmic reticulum. These phosphoinositide derivatives appear to promote calcium leak, contractile dysfunction, and protein catabolism. It remains to be seen whether PtdIns $(3,4) \mathrm{P} 2$ and PtdIns(3,5)P2 accumulate in the SR of aged muscles. This is an intriguing possibility. A detailed understanding of the cellular function of MIP/MTMR14 and the molecular events leading to saropenia may lead to clinical countermeasures to slow the age-related loss of muscular strength.

\section{REFERENCES}

1. Romero-Suarez $S$ et al. Aging. 2010; 2:404-413

2. Shen J et al. Nat Cell Biol. 2009; 11:769-776. 